\title{
Assessment of self care practice in patients with diabetes mellitus in tertiary care teaching hospital
}

\author{
Patel $\mathrm{DA}^{1,}$ Solanki $\mathrm{MN}^{1 *}$, Desai $\mathrm{CK}^{1}$, Parmar $\mathrm{KG}^{2}$
}

1. Department of Pharmacology, BJ Medical College, Ahmedabad

2. Department of Medicine, Civil Hospital Ahmedabad

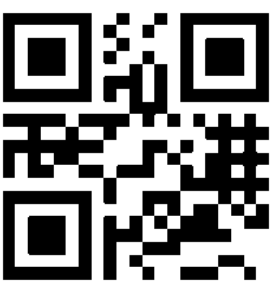

Abstract :Background: Diabetes is one the global health emergencies of the $21^{\text {st }}$ century with a prevalence of $9.1 \%$ and accounts for 5 million deaths annually. Selfcare practices in diabetes patients along with medicines are crucial to keep the illness under control and prevent complications. Effective management of diabetes will be a difficult task without an adequate understanding of the existing level of practice related to diabetes self-care. Objective: To assess the level of self care recommendation and self care practice among the patients of diabetes mellitus. Methods: This was a cross-sectional, questionnaire-based, single center study carried out in patients of Diabetes Mellitus type 1 and type 2 who met the inclusion and exclusion criteria. Patients were evaluated for self-care recommendations and self-care practice comprising of different components like diet, exercise, blood sugar testing, foot care, medication, and smoking by using SDSCA (Summary of Diabetes Self Care Activities) questionnaire. The number of patients achieve $>50 \%$ of SDSCA score for different components as well as a total SDSCA score were analyzed. Gender wise comparison of different components and total scores were done by applying unpaired t-test $(p<0.05)$. Results: A total of 88

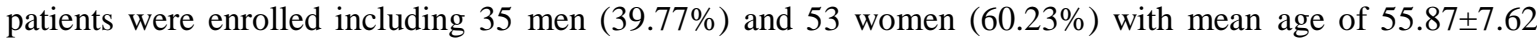
years. Self care recommendation was received by all patients. Total score of study population ranges from 33 to 93 with mean score $66.26 \pm 0.97$. Out of 88 patients, $92 \%$ patients obtained $>50 \%$ of total score (total score $=113$ ). $55.7 \%$ patients achieved $>50 \%$ score for diet while $68.2 \%, 63.6 \%, 84.1 \%$, and $90.9 \%$ patients achieved $>50 \%$ of total SDSCA score for exercise, blood sugar testing, foot care practice and medication respectively. Majority of patients $(84.1 \%)$ do not smoke. Foot care practice were found significantly higher in men than women amongst all components $(p<0.023)$. Conclusion: Level of self-care recommendation and practices were found to be adequate. More efforts should be put to encourage diabetic patient implement these self care practices to prevent complications and to have better quality of life.

Key words: Self care, SDSCA, Diabetes Mellitus

*Corresponding Author

Dr. Manish Solanki

E-mail id:siddharthpanchal22@gmail.com

Copyright: This is an open-access article distributed under the terms of the Creative Commons Attribution license which permits unrestricted use, distribution, and reproduction in any medium for non-commercial use (Non Commercial, or CC-BY-NC) provided the original author and source are credited.

\section{INTRODUCTION}

Diabetes is one of the global health emergencies of the $21 \mathrm{st}$ century with a prevalence of $9.1 \%$ and accounts for 5 million deaths annually. Amongst the non communicable disease diabetes considered to be the most markedly seen in middle or low income countries. ${ }^{1} \mathrm{As}$ per the International Diabetes Federation (IDF), South-East Asia and Western Pacific regions are at the epicenter of the diabetes crisis: China alone has 121 million people with diabetes and India's diabetes population totals 74 million and it is predicted that this figure will cross 100 million by $2030 .^{2}$ Management of diabetes includes pharmacological and non pharmacological measures. With the help of pharmacological management we can control blood sugar level, increase insulin secretion and also helps to prevent the long term damage, dysfunction, and failure of various organs which otherwise leads to increased morbidity and mortality ${ }^{3}$ but the only problem with medication is that we cannot cure the whole condition and also there are side effects of hypoglycemic drugs like, nausea, vomiting, abdominal pain etc. We can only prevent the damage so with the help of non pharmacological management like lifestyle modification and self care practice, the patient have a better quality of life. Self care in diabetes is an evolutionary process of development of knowledge or awareness by learning to survive with the complex nature of diabetes in a social context. ${ }^{4}$ Self care of diabetes is essential for control of disease and improvement in quality of life of patient. 
Diabetes self care activities are behaviors undertaken by people with or at risk of diabetes in order to successfully manage the disease on their own. ${ }^{5}$ However, research indicates that $50 \%$ to $80 \%$ of persons living with diabetes worldwide have substantial knowledge deficits regarding the management of their condition. ${ }^{6}$ Diabetes self-management education is defined as an ongoing process of facilitating the knowledge, skills, and ability necessary for diabetes self-care. Diabetes self management education is a critical element of care for all people with diabetes and is necessary in order to improve patient outcomes. ${ }^{7}$ Because most patients living with diabetes receive regular care in primary-care settings, it is imperative that resources are allocated and diabetes selfmanagement education systems are developed specifically for primary-care practices. ${ }^{8}$ No ideal educational program exists however, several guidelines have been published by the American Diabetes Association, the American Association of Diabetes Educators, the International Diabetes Federation, and the National Institute for Clinical Excellence indicating how diabetes self management education should be implemented. ${ }^{9}$ There is growing concern regarding Diabetes mellitus with reported high prevalence. In the Indian sociocultural scenario, it has been reported that adherence to treatment regimens is very poor due to poor attitude towards the disease and poor health literacy. As the prevalence increases, along with medical and pharmaceutical development, human factor advancement educational programs need to increase their effectiveness. Adequate baseline information about the prevalence of good self care activities is not available from India. ${ }^{10}$ Self care management will improve blood sugar control and also helps to prevent further complications. So the present study was conducted to analyse the extent and pattern of self care practice as a part of patient education programme in the diabetic patients.

\section{MATERIALS AND METHODS}

This was an observational, cross sectional, single center study carried out to assess the self care practice in patients with diabetes mellitus in tertiary care teaching hospital at outdoor patient Department of Medicine, Civil hospital, Ahmedabad located in Gujarat state for 2 months (March 2019 to May 2019). Prior approval from institutional ethics committee was taken. Investigator attended the diabetic OPD twice a week, patients of either gender and 18 years to 80 years of age who visited OPD with diabetes mellitus type 1 or type 2 and given written informed consent were enrolled in the study whereas patients with less than 1 year duration of diabetes mellitus were excluded.

SDSCA (Summary of Diabetes Self Care Activities),Probably the most widely used selfreport instrument for measuring diabetes selfmanagement in adults was administered to the enrolled patients. ${ }^{11}$ SDSCA questionnaire (a revised version of the SDSCA) a standardized, validated questionnaire was used to assess selfcare activities of diabetic patients. Translation of questionnaire was done in two languages Hindi and Gujarati. SDSCA is a brief 6 item questionnaire consisting of general diet, specific diet, physical activity, blood glucose testing, foot care, medication and one item for smoking measuring the number of days of the previous 7 days during which the patient has reported adequate adherence to self-care activities. Patient who provided incomplete/inadequate information was excluded.

Data analysis: The collected data were entered in MS Excel, and analysed in the form of percentage, mean and standard error of mean (SEM) and statistical test like unpaired t test were applied and $p<0.05$ is considered as statistically significant difference. Analysis was done on the following parameters,

a) Number of patients received self care recommendation

b) SDSCA score of study population

i) Component wise score in study population ii) Total score of all the components iii) Gender wise comparison of SDSCA score

\section{RESULTS}

Among 88 patients enrolled in this study, 35 patients were men $(39.77 \%)$ and 53 were women $(60.23 \%)$ with mean age of $55.87 \pm 7.62$ years. Self care recommendation was received by all patients $(n=88)$. When SDSCA component wise score was analysed on the basis $>50 \%$ or $<50 \%$ score achieved by individual patient, it was found that majority of the patients achieved $>50 \%$ of score for each SDSCA component as shown in figure 1.

Out of total 88 patients, $77 \%$ patients for diet, $68.20 \%$ patients for exercise, $59.10 \%$ patients for foot care, $63.63 \%$ patients for blood sugar testing and $90.91 \%$ patients for medication achieved $>50 \%$ of SDSCA component wise score. Smoking was done by $15.9 \%$ of patients. 


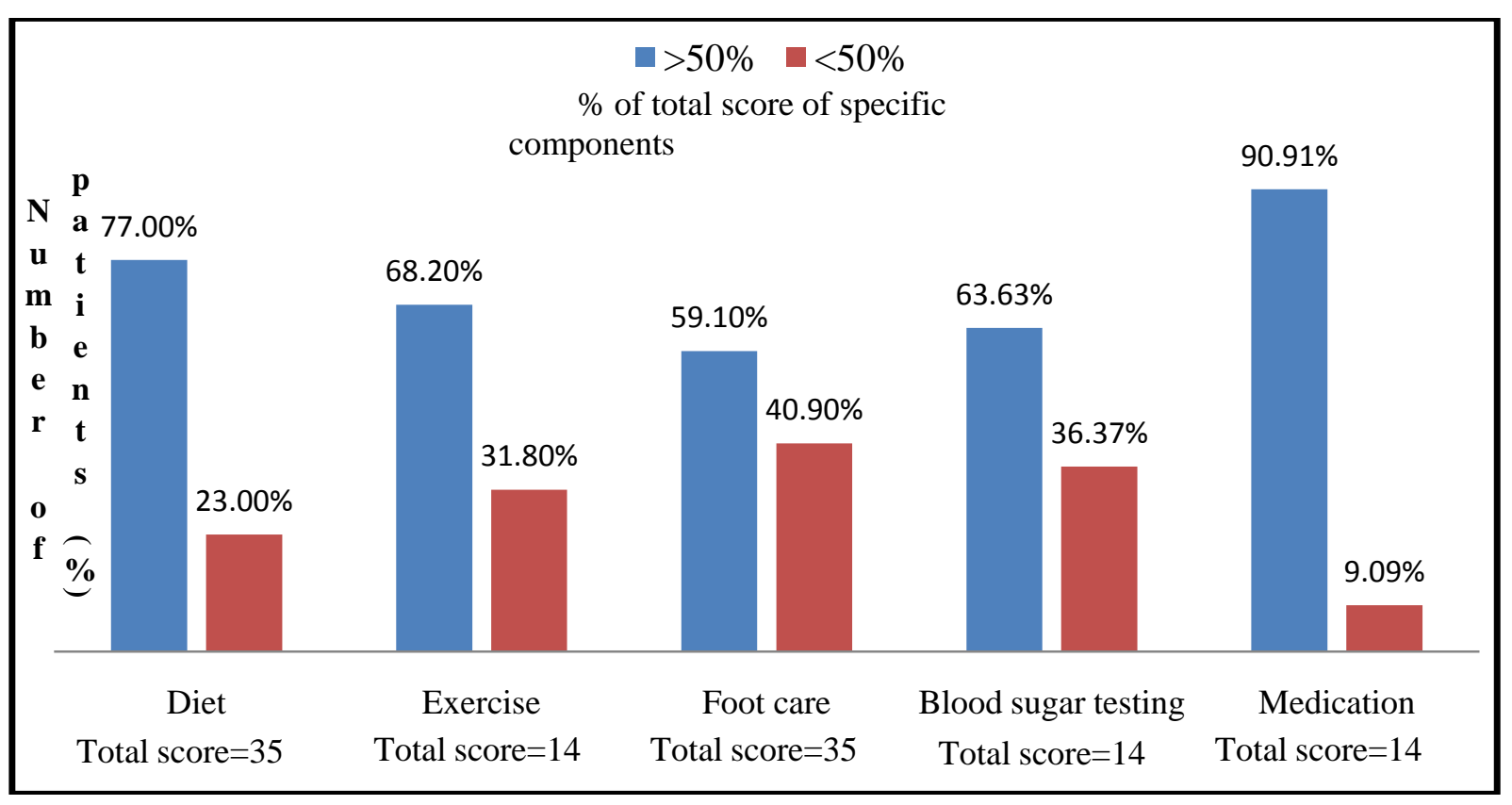

Figure 1: SDSCA component wise distribution of study population $(\mathrm{n}=\mathbf{8 8})$

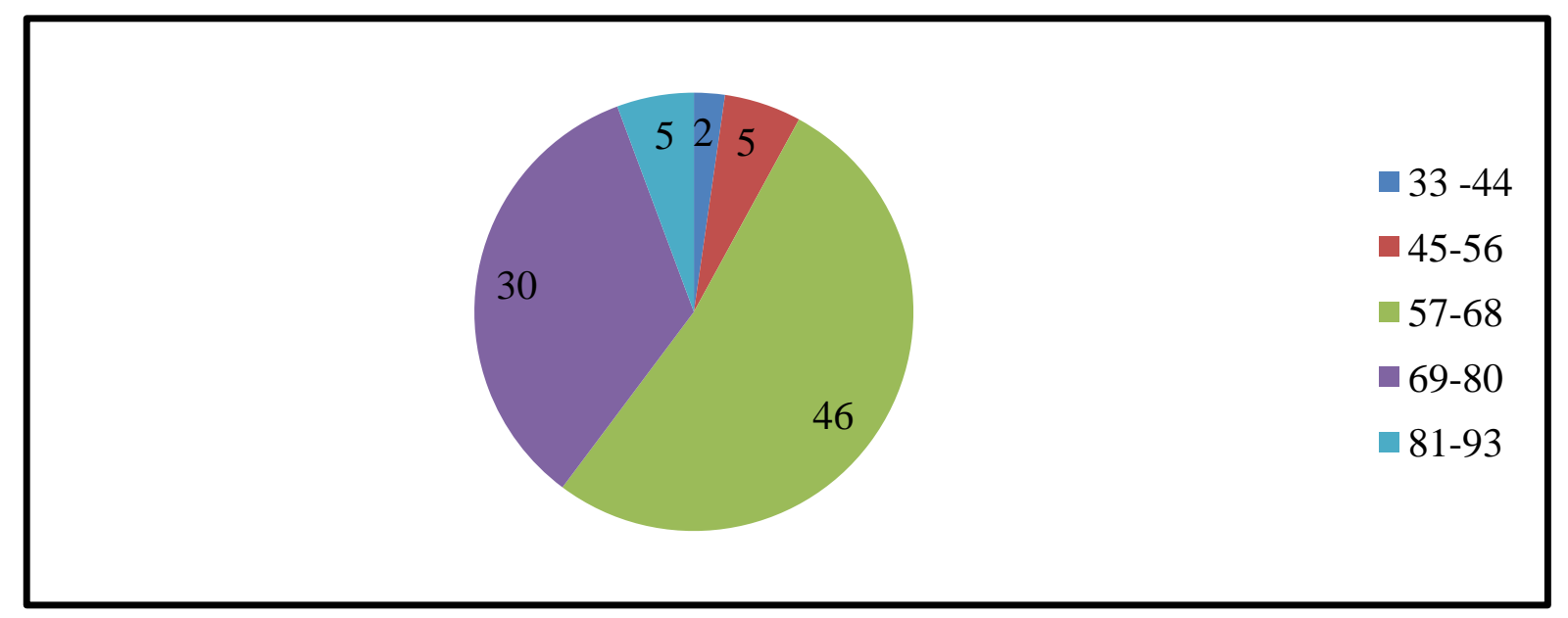

Figure 2: SDSCA total score wise distribution of study population $(\mathrm{n}=\mathbf{8 8})$

Total score of the SDSCA questionnaire is 113 (diet: 35, exercise: 14, blood sugar testing: 14, medication: 14 , foot care practice: 35 , smoking: 1). SDSCA Total score of study population was found in the range of 33 to 93 with $66.26 \pm 0.97$ (mean \pm SEM). Out of 88 patients, 46 patients scored between 57-68 and 30 patients scored between 69-80. While five patients achieved highest score between $81-93$ and only 2 patients achieved lowest score of 33-44,hence it is found that majority of patients $(86.4 \%, \mathrm{n}=88)$ achieved more than $50 \%$ of SDSCA total score. (Figure: 2 ).

On further analysis of study population, SDSCA total score was found $65.11 \pm 0.25$ in women and $68 \pm$ 0.30 in men and no significant difference was found between them. When SDSCA specific component wise score between men and women was compared, no significant difference was found in components like diet, exercise, blood sugar testing, smoking and medication. However score for foot care practice was found significantly higher in men than in women $(p<0.023)$ which is shown in table 1. 
higher in men than in women, less attentive nature of women regarding foot care might be the reason for the difference. Foot care practices were also lacking among the study participants. In the study done by Rao NS, ${ }^{19}$ it was found that foot care practices were lacking in $56.5 \%$ of the study participants and only $10.1 \%$ of the study participants practiced optimum foot care regularly. Raithatha SJ et al, study reported only 9\% patients checking their feet on a routine basis for any damage to the skin. Foot care is important because of long duration of diabetes causes neuropathy and diabetic foot damage with the help of regular self care we can control the damage. Blood sugar monitoring was seen high (71\%) in D Rajeshkaran et al, 2015 whereas it was found $(63.63 \%)$ in our study, Similar results have been reported in other community based studies in Maharashtra, (Kushwaha AS,et al), ${ }^{16}$ Gujarat, and Andhra Pradesh study done by Rao $\mathrm{NS}$, et al ${ }^{19}$ with self monitoring of blood glucose level lacking in $70 \%, 84 \%$, and $93.3 \%$ of the study participants, respectively. Main reason for lack in blood sugar testing was lower socioeconomic class as they could not afford a glucometer and its strips and also it might be due to fear of needle puncture. Contrasting results have been shown by other studies from Vellore, Bengaluru, and Mangalorewith the very high prevalence of regular monitoring of blood sugar (70\%, $76.6 \%$ and $73 \%$ respectively) however their criteria for regular blood sugar monitoring was different which was once a month or once 3 monthly. Diabetic patients who were put on insulin were generally more aware of the need to carry out blood sugar testing because of advice received from doctors while they were taught self injections with insulin and their own experience of hypoglycemic episodes. Similarly, many younger diabetics had a fear of debilitating long term complications which caused due to increase or decrease in glucose level which made them responsible for carrying out blood sugar testing to prevent it.About $15.9 \%$ patients reported as having history of smoking. Much higher prevalence of smoking among diabetic patients as compared to our study has been observed in studies conducted by V. Gopicharan, with the prevalence of $43.4 \%$. There may be a possibility of patients hiding their smoking status which may be a reason for the low prevalence of smoking found in our study compared to other studies. The absence of family support had a negative effect on self care activity for diabetic patients, it is well known that family members can interfere with or facilitate self care activities (e.g., by buying groceries) and thereby contributing to diabetes control among patients. ${ }^{20}$ The limitation of our study is that it was conducted at tertiary care teaching hospital ahmedabad, India and therefore, the findings of our study are generalizable only to this area and may not be applicable to the whole of diabetic patients. Therefore external validity may be lacking. Since SDSCA is a self report questionnaire, and our study is cross sectional there are issues regarding the temporality and subjective answers therefore cause and effect relationship may not be clear in this study.

\section{CONCLUSION}

Self-care recommendations is given to all patients, while overall level of self-care activities among diabetic patients in our study is adequate, however there is a need for ongoing self management education programs in all hospitals, for patients as well as care givers and also further study involving the objective measurement of self care activities is recommended.

Conflicts of interest: Declared, authours have no conflict of interest

\section{Funding/Financial support:None \\ REFERENCES}

1. International Diabetes Federation. IDF Diabetes Atlas. 7th ed. Brussels, Belgium: International Diabetes Federation; 2015. Available

from: http://www.diabetesatlas.org. [Last accessed on 2019 SEP 30].

2. Mathers CD, Loncar D. Projections of global mortality and burden of disease from 2002 to 2030. PLoS Med 2006;3:e442.

3. World Health Organization. Definition, Diagnosis and Classification of Diabetes Mellitus and its Complications. Report of a WHO Consultation. Geneva: World Health Organization; 1999.

4. Cooper HC, Booth K, Gill G. Patients' perspectives on diabetes health care education. Health Educ Res 2003;18:191- 206.

5. American Association of Diabetes Educators.AADE7 self care behaviors. Diabetes Educ 2008;34:445-9.

6. Strine TW, Okoro CA, Chapman DP, et al: The impact of formal diabetes education on preventive health practices and behaviors of persons with type 2 diabetes. Prev med 41P:79,2005.

7. MM Funnell, TL Brown, BP Childs, LB Hass.et al. national standard for diabetes self management education. Diabetes care 32 (supplement 1), S87-S94, 2009.

8. SIMINERIO LM: The diabetes education renaissance. Diabetes spectrum 19: 276, 2013. 
9. Formosa C, Vella L: Influence of diabetes related knowledge on foot ulceration. J diabetes Nurs 16: 111, 2012.

10. Toobert DJ, Hampson SE, Glasgow RE. The summary of diabetes self care activities measure: result from 7 studies and revised scale. Diabetes care. 2002; 25(7):1159-71.

11. NATIONAL INSTITUTE FOR CLINICAL EXCELLENCE: Type 2 diabetes: NICE guidelines [CG66]. Available at: http ://www.nice.org.uk/nicemedia/pdf/cg66nice guideline pdf. Published May 2008. Accessed Sep 2019

12. SHRIVASTAVA SR, SHRIVASTAVA PS, RAMASAMY J: Role of selfcare in management of diabetes mellitus. J Diabetes Metab Disord 12: 14, 2013.

13. FORMOSA C, LUCAS K, MANDY A, ET AL: Influence of national culture on diabetes education in Malta: a case example. Diabetes Primary Care 10: 109, 2008.

14. V Gopicharan, S Lyndon, MK angel et al: Diabetes self care activities:a community based survey in urban southern india National medical journal of India

15. Padma K, Bele DS, Bodhare TN, evaluation of knowledge and self care practices in diabetic patients and their role in disease management. Natl J community Med.2012;3;3-6.

16. Kushwaha AS, Kumari S, Kushwaha N. Self care in diabetes: A study amongst diabetics in an urban community. Int $\mathbf{J}$ Community Med Public Health 2016;3:293- 8.

17. Raithatha SJ, Shankar SU, Dinesh K. Self- care practices among diabetic patients in Anand district of Gujarat. ISRN Family Med 2014;2014:743791.

18. Rao NS, Krishna YB. Knowledge and practice among type 2 diabetes patients from a village in Guntur district of Andhra Pradesh. Int J Health Sci Res 2014;4:6- 12.

19. Sasi ST, Kodali M, Burra KC, Muppala BS, Gutta P, Bethanbhatla MK, et al. Self care activities, diabetic distress and other factors which affected the glycaemic control in a tertiary care teaching hospital in South India. J Clin Diagn Res 2013;7:857-60.

20. Fisher L, Chesla CA, Skaff MM, Gilliss C, Mullan JT, Bartz RJ, et al. The family and disease management in hispanic and European-American patients with type 2 diabetes. Diabetes Care 2000;23:267,72. 